\title{
Le caryotype du porc villageois de Papaousie Nouvelle-Guinée
}

\author{
C.P. POPESCU *, J.J. LAUVERGNE ** et G.L. MALYNICZ *** \\ I.N.R.A., * Laboratoire de Cytogénétique \\ ** Laboratoire de Génétique factorielle \\ Centre de Recherches zootechniques, F 78350 Jouy-en-Josas \\ *** Department of Primary Industry, Livestock Branch, P.O. Box 417 \\ Konedobu, Papouasie Nouvelle-Guinée
}

\begin{abstract}
Résumé
Le caryotype de 13 porcs domestiques indigènes de Papouasie Nouvelle-Guinée s'est révélé tout à fait semblable à celui des porcs domestiques européens : $2 n=38$ chromosomes ; 12 paires de méta ou submétacentriques, 6 paires d'acrocentriques, un $X$ submétacentrique et un petit $\mathrm{Y}$ submétacentrique. Une origine commune à ces deux populations domestiques est donc possible.
\end{abstract}

Le porc est le principal animal domestique de Papouasie Nouvelle-Guinée. Ses caractéristiques zootechniques et son système d'élevage ont été étudiés : voir CoOPER et al., 1981, pour revue. Par contre, jusqu’à présent, son caryotype était inconnu.

Pour remédier à cette lacune, des échantillons de sang ont été prélevés sur 15 animaux en août 1981 dans le troupeau expérimental du Tropical Pig Breeding and Research Center (T.P.B.R.C.) de Goroka (Eastern Highlands Province, E.H.P.), établissement de recherche dépendant du Department of Primary Industry (D.P.I.) de Papouasie Nouvelle-Guinée ainsi que dans le village de Lapegu, situé à $10 \mathrm{~km}$ de Goroka. Les caractéristiques (âge, sexe, coloration) de ces 15 porcs sont données dans le tableau 1. Les animaux de l'échantillon étaient considérés par les techniciens du D.P.I. de Goroka comme de pure race autochtone. Cela est confirmé par l'examen des colorations données dans le tableau 1. Les proportions des différents patrons de coloration sont analogues à celles des populations indigènes, exemptes d'infusion de sang exotique, considérées par LAUVERGNE et al. (1982).

Les analyses ont été faites par la méthode de culture de sang intégral au Laboratoire de Cytogénétique du Département de Génétique animale de l'I.N.R.A. Pour 
13 animaux (9 du T.P.B.R.C. et 4 de Lapegu), les cultures ont réussi. Les chromosomes ont alors été colorés au Giemsa et plusieurs caryotypes dressés pour chaque animal.

Les 13 animaux examinés présentent tous le même caryotype à 38 chromosomes : 12 paires d'autosomes méta ou submétacentriques, 6 paires d'autosomes acrocentriques, 1 chromosome $\mathrm{X}$ submétacentrique et 1 petit chromosome $\mathrm{Y}$ également submétacentrique (fig. 1).

\section{Tableau 1}

Caractéristiques des porcs Papous indigènes de l'étude.

Caracteristics of the native Papuan pigs of the study.

\begin{tabular}{|c|c|c|c|c|}
\hline & $\begin{array}{l}\text { Sexe } \\
\text { Sex }\end{array}$ & $\begin{array}{l}\text { Age } \\
\text { Age }\end{array}$ & $\begin{array}{c}\text { Coloration } \\
\text { Colour pattern }\end{array}$ & $\begin{array}{l}\text { Provenance } \\
\text { Source }\end{array}$ \\
\hline 1 & $\mathbf{F}$ & porcelet & agouti & Lapegu (E.H.P.) \\
\hline $2(1)$ & $\mathbf{M}$ & porcelet & noir & Lapegu (E.H.P.) \\
\hline 3 & $\mathbf{M}$ & porcelet & noir & Lapegu (E.H.P.) \\
\hline 4 & $\mathbf{M}$ & adulte & noir & Lapegu (E.H.P.) \\
\hline 5 & $\mathbf{M}$ & adulte & domino & Lapegu (E.H.P.) \\
\hline 6 & $\mathbf{F}$ & porcelet & noir & $\begin{array}{l}\text { T.P.B.R.C. (Goroka) } \\
\text { (E.H.P.) }\end{array}$ \\
\hline $7(1)$ & F & porcelet & domino & $\begin{array}{l}\text { T.P.B.R.C. (Goroka) } \\
\text { (E.H.P.) }\end{array}$ \\
\hline 8 & $\mathbf{F}$ & porcelet & domino & $\begin{array}{l}\text { T.P.B.R.C. (Goroka) } \\
\text { (E.H.P.) }\end{array}$ \\
\hline 9 & $\mathbf{M}$ & porcelet & domino & $\begin{array}{l}\text { T.P.B.R.C. (Goroka) } \\
\text { (E.H.P.) }\end{array}$ \\
\hline 10 & $\mathbf{M}$ & porcelet & noir & $\begin{array}{l}\text { T.P.B.R.C. (Goroka) } \\
\text { (E.H.P.) }\end{array}$ \\
\hline 11 & $\mathbf{F}$ & porcelet & domino & $\begin{array}{l}\text { T.P.B.R.C. (Goroka) } \\
\text { (E.H.P.) }\end{array}$ \\
\hline 12 & $\mathbf{F}$ & adulte & domino brun? (2) & $\begin{array}{l}\text { T.P.B.R.C. (Goroka) } \\
\text { (E.H.P.) }\end{array}$ \\
\hline 13 & $\mathbf{F}$ & adulte & noir & $\begin{array}{l}\text { T.P.B.R.C. (Goroka) } \\
\text { (E.H.P.) }\end{array}$ \\
\hline 14 & $\mathbf{M}$ & adulte & noir & $\begin{array}{l}\text { T.P.B.R.C. (Goroka) } \\
\text { (E.H.P.) }\end{array}$ \\
\hline 15 & $\mathbf{M}$ & adulte & domino & $\begin{array}{l}\text { T.P.B.R.C. (Goroka) } \\
\text { (E.H.P.) }\end{array}$ \\
\hline
\end{tabular}

(1) Animal pour lequel la culture de sang n'a pas réussi.

(2) Phénotype coloré non définitivement précisable, en tout cas différent de tous ceux des races exotiques. 

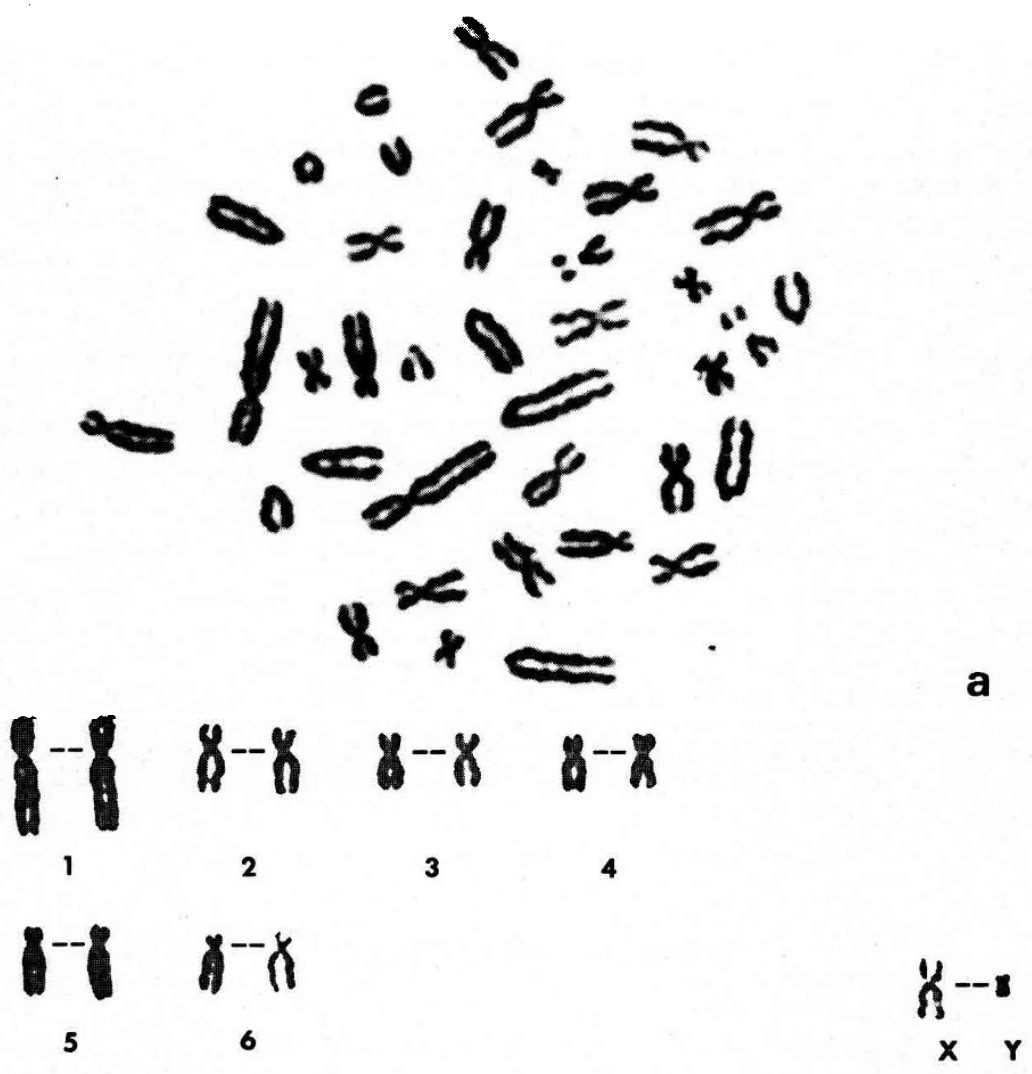

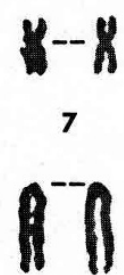

13<smiles>[AlH2]</smiles>

8

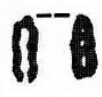

14

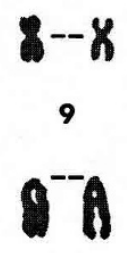

15

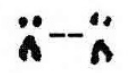

10

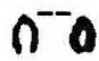

16 a

$$
\text { หา- } x-x
$$

11

12

$a^{-a}$

$0-0$

$18 \quad b$

Métaphase (a) et caryotype (b) du porc mâle indigène de Papaousie Nouvelle-Guinée. Metaphase (a) and karyotype (b) of the male native pig of Papua New-Guinea.

Cette configuration est tout à fait semblable à celle du caryotype des porcs domestiques européens, selon le modèle standardisé proposé par HANSEN (1977).

Cette ressemblance caryotypique permet de penser que le porc domestique indigène de Papouasie Nouvelle-Guinée et le porc domestique d'Europe pourraient avoir la même origine ancestrale. 


\section{Remerciements}

Cette recherche a été effectuée dans le cadre d'un programme de coopération France Papaousie Nouvelle-Guinée, pour l'étude et le développement de l'élevage du porc en Papouasie Nouvelle-Guinée.

Nous tenons à remercier R. Nelson du Veterinary Service (D.P.I.) de Goroka, Chris Rose et Mangiri Kipo du T.P.B.R.C. (Goroka), ainsi que les habitants du village de Lapegu.

La note a été relue par P. Sellier, Département de Génétique de l'I.N.R.A.

\section{Summary}

The karyotype of the village pig of Papua New-Guinea

The karyotype of 13 village pigs of Papua New-Guinea was found very similar with that of domestic european ones : $2 \mathrm{n}=38$ chromosomes; 12 pairs of meta or submetacentrics, 6 pairs of acrocentrics, a submetacentric $X$ and a small submetacentric $Y$. A common origin for the european domestic and the papuan domestic pigs is therefore possible.

\section{Références bibliographiques}

Cooper Ann B., Lauvergne J.J., Malynicz G.L., Quartermain A.R., 1981. Pig Husbandry in Papua New-Guinea : a bibliography. Ann. Génét. Sél. anim., 13, 301-330.

HANSEN K.M., 1977. Identification of the chromosome of the domestic pig (Sus scrofa domesticus). An identification key and landmark system. Ann. Génét. Sél. anim., 9, 517-526.

Lauvergne J.J., Malynicz G.L., Quartermain A.R., 1982. Coat colour variants of village pigs in Pupa New-Guinea. Ann. Génét. Sél. anim., 14, 49-62. 